\title{
Rescue PCI Immediately after Failed Primary CABG in Patient with Cardiogenic Shock
}

\section{Mohammed Habib ${ }^{1 *}$ and Merwan Sadek ${ }^{2}$}

${ }^{1}$ Head of Cardiac Catheterization Department, Public Aid Hospital, Israel

${ }^{2}$ Head of Cardiac Surgery Department, Public Aid Hospital, Israel

Keywords: Coronary Artery Bypass Grafting (CABG); ST-Elevation Myocardial Infarction (STEMI); Cardiogenic shock; Angiography

\section{Introduction}

Perioperative ischemia remainsa significant problem and carries a very high morbidity and mortality in patients undergoing coronary artery bypass grafting $(\mathrm{CABG})$ in patients with ST-elevation myocardial infarction (STEMI). Different therapeutic options are available for the clinician to manage this complication. These include direct reoperation, percutaneous coronary intervention (PCI) and conservative medical management. Immediate PCI has been reported to be a viable alternative to emergent redo $\mathrm{CABG}$ in these patients. Here in, we report an additional case of emergent PCI immediately after failed primary $\mathrm{CABG}$ in patient with cardiogenic shock.

\section{Case Report}

A 70-year-old male patient diabetic, hypertensive, presents with angina pectoris on mild effortdespite optimal medical treatment, patient was transfereeto our cardiac catheterization unit and Coronary angiography was performed (Figures 1 and 2).

After 10 dayscardiac surgery planned, just before Cardiac surgery chest pain was developed, the ECG: SR, ST elevation in inferior leads. Blood pressure was: 50/30 mmHg. Norepinephrine $10 \mathrm{mcg} / \mathrm{kg} / \mathrm{min}$ was started and intraaortic balloon pump was inserted, afterthatthe surgeon was started the operation as isolated primary CABG.

Surgical revascularization was performed in the patients using median sternotomy, standard cardiopulmonary bypass (CPB) technique with ascending aortic and two stage venous cannulation, mild hypothermia and cold crystalloid cardioplegic arrest. Heparin was administered to achieve an activated coagulation time exceeding 400 seconds. Left Internal thoracic artery, radial artery, and saphenous vein grafts, were used as graft conduits (LIMA to LAD, SVG to Diagonal branch and SVG to OM branch). Protamine was administered to reverse heparin according to standard practice. Patient was transferred tointensive care unit.

ECG, Blood Gases, Invasive blood pressure, CBC were determined

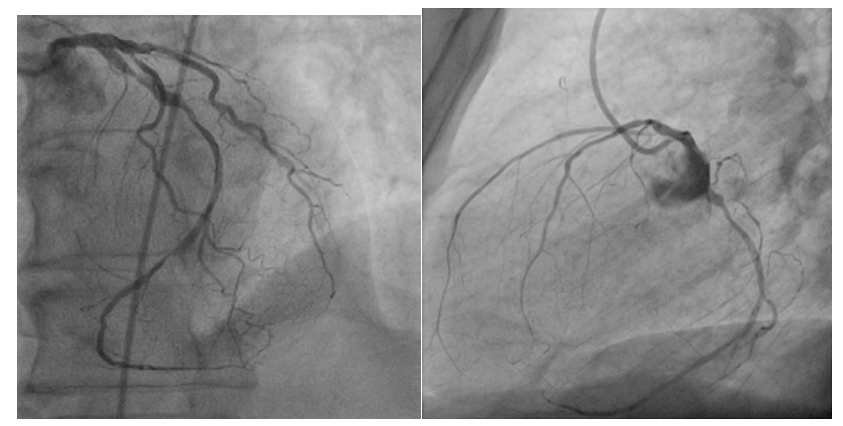

Figure 1: $A$ and $B$ left coronary angiography: LM: normal, LCX proximal and distal tight lesions, LAD proximal severe stenosis and diagonal branch proximal severe stenosis. immediately after arrival on the intensive care unit, BP: $60 / 35 \mathrm{mmHg}$, ECG: sinus tachycardia, ventricular premature contraction, inferior ST elevation. PH:6.8, HGb:11.5. The patient was transfer urgently to coronary angiography.

Urgent coronary angiography suggested that: totally occluded in proximal LCX (Figure 3).

Predilatation with $1.5 \times 15 \mathrm{~mm}$ sapphireballoon in proximal and distal LCX was done then 3 Bare Metal integritystents $(3 \times 15 \mathrm{~mm}$, $2.75 \times 12 \mathrm{~mm}$ and $2.75 \times 18 \mathrm{~mm}$ stent) were deployed in proximal and distal LCX. Thrombus was seen in distal LCX, intracoronary Glycoprotein IIB/IIIa Inhibitor (aggrastat) $20 \mathrm{ml}$ slowly was infused in the left coronary artery via guiding catheter with good result (Figure 4).

The BP increase up to $125 / 70 \mathrm{mmHg}$, and VPCs were resolved, ST elevation normalized, after 72 hours patient was extubated. We started antiagregant aspirin $100 \mathrm{mg}$ tablet daily after 24 hours of operation. He was eventually discharged home in stable condition, 12 days after the conclusion of the procedure. The treatment on discharge were aspirin $100 \mathrm{mg}$ one daily, clopidogrel $75 \mathrm{mg}$ once daily, atorvastation $80 \mathrm{mg}$ once daily, ramipril $5 \mathrm{mg}$ once daily and bisoprolol $5 \mathrm{mg}$ one daily. After 3 weeks echocardiography suggest e.g. preserved LV systolic function, EF:55\%, and mild mitral regurgitation.

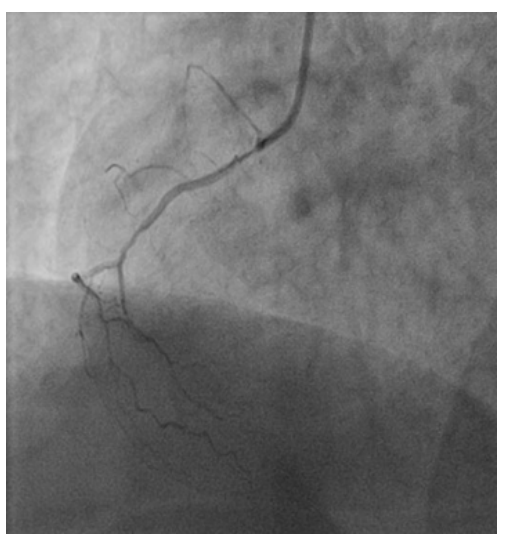

Figure 2: RCA small and diseased

*Corresponding author: Mohammed Habib, Head of Cardiac Catheterization Department, Public Aid Hospital, Israel, Tel: 28026 24; E-mail: cardiomohammad@yahoo.com

Received October 07, 2013; Accepted February 14, 2014; Published February 16,2014

Citation: Habib M, Sadek M (2014) Rescue PCI Immediately after Failed Primary CABG in Patient with Cardiogenic Shock. J Clin Case Rep 4: 345. doi:10.4172/21657920.1000345

Copyright: @ 2014 Habib M, et al. This is an open-access article distributed under the terms of the Creative Commons Attribution License, which permits unrestricted use, distribution, and reproduction in any medium, provided the original author and source are credited. 


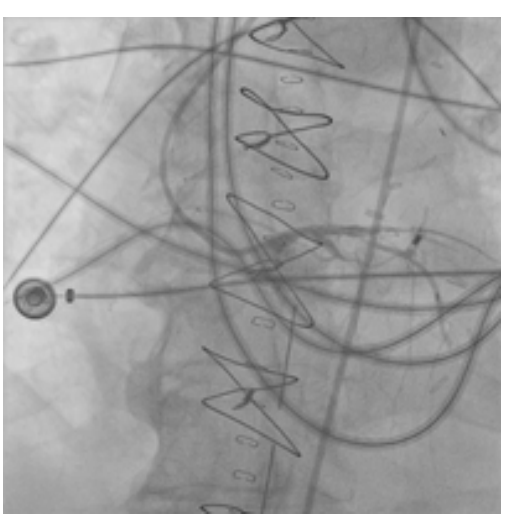

Figure 3: Angiography post CABG: LCX Proximal totally occluded. Intra-Aortic Balloon was seen in abdominal aorta.

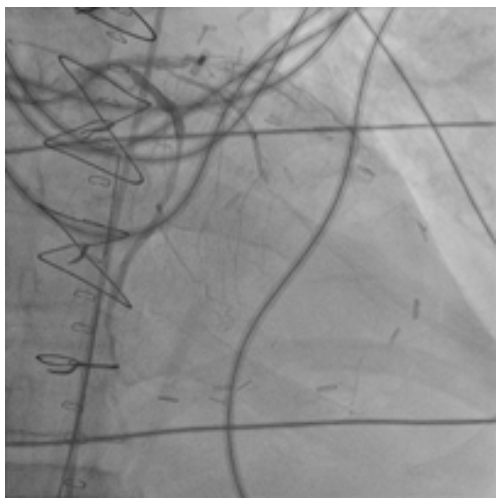

Figure 4: LCX after 3 stents deployment in LCX.

\section{Discussion}

Perioperative myocardial ischemia is rare but serious complication of CABG. Incorrect graft anastomosis, graft spasm, displacement and dissection, coronary artery thrombosis and incomplete revascularization are main causes [1]

Although it has been shown that patients undergoing CABG due to
AMI have a significantly higher risk of dying in the hospital, and there are many data on the outcomes of CABG with acute, evolving, ongoing, orprevious $\mathrm{MI}$ in general $[2,3]$.

Primary CABG in STEMI patients areassociated with significantly higher in-hospital mortality and a higher incidence of adverse events, In-hospital mortality rates clearly varied, however, depending on the time interval from symptom onset to time of operation, with $10.8 \%$ for patients undergoing CABG within 6 hours after onset of symptoms, rising to $23.8 \%$ mortality within 7 to 24 hours after symptom onset, declining to $6.7 \%$ mortality after 1 to 3 days, and $4.2 \%$ after 4 to 7 days, and finally $2.4 \%$ after 8 to 14 days [4].

Recent study suggested that, overall in hospital mortality in patient withurgent PCI immediately after failed CABG was 30\% [1].

In our case we started intropic treatment, intra-aortic counter pulsation and immediate coronary angiography and PCI with infusion intracoronary of GPIIb/IIIa inhibitors. This is the first case reported of successful rescue PCI immediately after primary CABG in patient with cardiogenic shock.

\section{Conclusion}

Perioperative ischemia is rare complication of CABG. Coronary angiography is safe and effective in determining the cause of myocardial ischemia and treatment strategy. No randomized studies confirming superiority of invasive strategy. However, PCI seems to be safe and successful treatment completing myocardial revascularization. Stenting of coronary arteries is usually preferred approach probably because it is easier and with fewer complications.

\section{References}

1. Szavits-Nossan J, Stipić H, Sesto I, Kapov-Svilicić K, Sipić T, et al. (2012) Angiographic control and percutaneous treatment of myocardial ischemia immediately after CABG. Coll Antropol 36: 1391-1394.

2. Lee DC, Oz MC, Weinberg AD, Ting W (2003) Appropriate timing of surgical intervention after transmural acute myocardial infarction. J Thorac Cardiovasc Surg 125: 115-119.

3. Albes JM, Gross M, Franke U, Wippermann J, Cohnert TU, et al. (2002) Revascularization during acute myocardial infarction: risks and benefits revisited. Ann Thorac Surg 74: 102-108.

4. Thielmann M, Neuhäuser M, Marr A, Herold U, Kamler M, et al. (2007) Predictors and outcomes of coronary artery bypass grafting in ST elevation myocardial infarction. Ann Thorac Surg 84: 17-24. 